\title{
Safety and Tolerability of Essential Oil from Cinnamomum zeylanicum Blume Leaves with Action on Oral Candidosis and Its Effect on the Physical Properties of the Acrylic Resin
}

\author{
Julyana de Araújo Oliveira, ${ }^{1}$ Ingrid Carla Guedes da Silva, ${ }^{1}$ \\ Leonardo Antunes Trindade, ${ }^{1}$ Edeltrudes Oliveira Lima, ${ }^{1}$ Hugo Lemes Carlo, ${ }^{1}$ \\ Alessandro Leite Cavalcanti, ${ }^{2}$ and Ricardo Dias de Castro ${ }^{1,2}$ \\ ${ }^{1}$ Post-Graduate Program in Dentistry, Federal University of Paraíba, Campus I, João Pessoa, PB, Brazil \\ ${ }^{2}$ Post-Graduate Program in Dentistry, State University of Paraíba, Campina Grande, PB, Brazil
}

Correspondence should be addressed to Ricardo Dias de Castro; ricardodiasdecastro@yahoo.com.br

Received 6 August 2014; Revised 28 October 2014; Accepted 13 November 2014; Published 9 December 2014

Academic Editor: Roberto K. N. Cuman

Copyright (C) 2014 Julyana de Araújo Oliveira et al. This is an open access article distributed under the Creative Commons Attribution License, which permits unrestricted use, distribution, and reproduction in any medium, provided the original work is properly cited.

\begin{abstract}
The anti-Candida activity of essential oil from Cinnamomum zeylanicum Blume, as well as its effect on the roughness and hardness of the acrylic resin used in dental prostheses, was assessed. The safety and tolerability of the test product were assessed through a phase I clinical trial involving users of removable dentures. Minimum inhibitory concentration (MIC) and minimum fungicidal concentrations (MFC) were determined against twelve Candida strains. Acrylic resin specimens were exposed to artificial saliva (GI), C. zeylanicum (GII), and nystatin (GIII) for 15 days. Data were submitted to ANOVA and Tukey posttest $(\alpha=5 \%)$. For the phase I clinical trial, 15 healthy patients used solution of C. zeylanicum at MIC (15 days, 3 times a day) and were submitted to clinical and mycological examinations. C. zeylanicum showed anti-Candida activity, with MIC $=625.0 \mu \mathrm{g} / \mathrm{mL}$ being equivalent to MFC. Nystatin caused greater increase in roughness and decreased the hardness of the material $(P<0.0001)$, with no significant differences between GI and GII. As regards the clinical trial, no adverse clinical signs were observed after intervention. The substance tested had a satisfactory level of safety and tolerability, supporting new advances involving the clinical use of essential oil from $C$. zeylanicum.
\end{abstract}

\section{Introduction}

Denture stomatitis is an inflammatory reaction in the oral tissues in contact with dentures and is often reported as being opportunistic infection, mainly caused by fungi of the genus Candida. In addition, they are more prevalent in patients using prostheses, immunosuppressed individuals, and chronic users of antimicrobial agents [1-3]. Clinically, it can be characterized as an erythematous area of well-defined contours, present in regions of contact between the dental prosthesis and oral cavity mucosa, causing changes in the texture of surfaces on which it develops $[4,5]$.
It is known that infection is related to contamination of the inner surface of the denture, since it is considered a reservoir of microorganisms due to irregularities and pores that develop on the surface of acrylic resin denture base materials [6-8]. In this context, the treatment of denture stomatitis lesions involves providing patients with guidance on denture and oral cavity cleaning, removal of potential irritant factors (poorly fitted and unsatisfactory dental prostheses), and use of antifungal agents and the manufacture of a new denture $[9,10]$.

However, it is known that this type of candidosis is not easy to treat, since relapses often occur after discontinuation 
of drug therapy, even if the traumatic factor has been eliminated by replacement with a new denture. Thus, treatment of this pathology is a challenge to clinical practice, given the intense frequency of drug administration, small number of drugs available, and increasing resistance expressed by microorganisms $[11,12]$.

Due to these undesirable factors and the relative toxicity present in conventional antimycotics, there is growing interest in the study of plants with therapeutic properties and antimicrobial activity, not only because they offer an alternative therapeutic approach, but also due to the perspective of isolating substances with significant efficacy against pathogenic fungi $[13,14]$.

One of the plant species recognized for its biological properties is Cinnamomum zeylanicum (cinnamon) [15]. Experimental in vitro and in vivo studies in animals and humans, conducted in different regions of the world, have shown numerous beneficial effects of C. zeylanicum on health [16]. Its analgesic, antiseptic, antispasmodic, astringent, insecticidal, and antimicrobial properties are among the main indications for its use [17-20]. These studies are linked to the idea of the low toxicity of extracts obtained from this plant, considering that it is used as food by human populations and that previous investigations in animals indicated no significant toxic effects, with wide therapeutic potential $[16,21]$, with reported hepatoprotective [22] and antioxidant effects $[23,24]$.

Among the properties required of materials used in the manufacture of dental prostheses, those related to surface roughness, surface tension, electrostatic interactions, and hardness are of clinical importance. According to the authors, surface roughness causes adhesion and retention of Candida albicans, which is of particular importance for the induction of stomatitis [25]. In this sense, it is relevant to determine the effects of antifungal agents on the acrylic resin used in the manufacture of dental prostheses, since the treatment protocol of oral candidiasis requires the product to be applied on the affected mucosa; thus it reaches the denture base.

Although there are reports on the antimicrobial activity of essential oils obtained from C. zeylanicum [26], clinical trials involving humans are scarce. Preclinical information can, however, be obtained from in vitro studies or with animal models; nevertheless, the first clinical tolerability data should also be obtained in humans [16]. The proposal of using C. zeylanicum in the treatment of oral candidiasis provides external use of the product (topical), which must be applied to affected oral mucosa without ingesting it, thus minimizing systemic effects. However, signs and symptoms resulting from the use of the product must be assessed by means of studies that include a phase I clinical study design. This generally includes healthy people in order to identify possible adverse effects, whereas phase II clinical studies, in turn, include unhealthy volunteers randomly distributed between test group and control group in order to obtain comparative efficacy data from a test substance and a standard substance, which has been well established in the literature [27].

Given the scarcity of studies addressing the antimicrobial properties of medicinal plants used in dentistry, in the present study, the action of essential oil from C. zeylanicum leaves was evaluated by assessing its antifungal activity and the possible changes caused by the test substance on the physical properties of heat-polymerized acrylic resin, which is the basic material of dentures. In a subsequent step, the study also sought to clinically investigate the safety and tolerability of the test product by means of a phase I clinical trial.

\section{Material and Methods}

2.1. Ethical Considerations and Trial Registration. The study was approved by the Research Ethics Committee of the Federal University of Paraíba and registered in the Brazilian Registry of Clinical Trials (http://www.ensaiosclinicos.gov.br/).

2.2. Study Design. This research was divided into three stages: an in vitro microbiological analysis of the antifungal activity of the test substance; effect of the test product on the physical properties of the acrylic resin; and phase I clinical trial investigating the safety and tolerability of the test product.

2.3. In Vitro Assays. Essential oil from C. zeylanicum leaves (cinnamon) (Ferquima Ind. e Com., Vargem Grande Paulista, São Paulo, Brazil) with characteristic appearance, color, odor, density, and refractive indexes $\left(20^{\circ} \mathrm{C}\right)$ of 1.043 and 1.533 , respectively, was used.

For chemical characterization of the test substance, chemical analysis by chromatography was performed using a gas chromatograph coupled to a mass spectrometer (Shimadzu GC-MS-QP5050A) and capillary column (J \& W SCIENTIFIC) with stationary phase of $5 \%$ phenyl and 95\% dimethylpolysiloxane, measuring $30 \mathrm{~m}$ long, $0.25 \mathrm{~mm}$ internal diameter, and $0.25 \mu \mathrm{m}$ of film thickness. The initial temperature programming ranged from $60^{\circ} \mathrm{C}$ to $240^{\circ} \mathrm{C}$ $\left(3^{\circ} \mathrm{C} / \mathrm{min}\right)$, while the programmed running time was 60 minutes, and the oven temperature was $250^{\circ} \mathrm{C}$. Helium was used as carrier gas (mobile phase) at a flow rate of $1.0 \mathrm{~mL} / \mathrm{min}$ with a 1:20 split ratio and injection volume of $1 \mu \mathrm{L}$. The compounds were identified by comparing their mass spectra with those existing in the database of the equipment (MIST Library, 2008). The essential oil sample was injected at a concentration of $2 \mathrm{ppm}$ and hexane was used as solvent. The chromatogram and mass spectra were analyzed with the aid of a device library and the integration parameters used were width: 3 and slope: 2000 .

A standard drug, antifungal nystatin (Sigma-Aldrich Brazil Ltda., São Paulo, Brazil), in the form of powder was used as positive control. The solutions were prepared at the time of performing the tests.

Step I: In Vitro Verification of the Antifungal Activity of Essential Oil from C. zeylanicum and Nystatin. Consider the following.

(I) Determination of the Minimum Inhibitory Concentration (MIC). MIC was determined using the microdilution technique [28] in 96-well plates that received $100 \mu \mathrm{L}$ of Sabouraud Dextrose Broth (Difco Laboratories, Detroit, Mich., USA). Then, $100 \mu \mathrm{L}$ of the test substance at an initial concentration of $5.000 \mu \mathrm{g} / \mathrm{mL}$ was distributed as follows: serial dilution was performed by collecting an aliquot of $100 \mu \mathrm{L}$ from 
a cavity containing a more concentrated substance to the following cavity. Sequentially, $10 \mu \mathrm{L}$ aliquots of the inoculum corresponding to 12 strains tested were dispensed in the holes of each column, 8 strains of Candida albicans, and 4 of Candida tropicalis (C. albicans ATCC 76485; C. albicans ATCC 76645; C. albicans LMP20; C. albicans LM111; C. albicans LM62; C. albicans LM108; C. albicans LM122; C. albicans LM86; C. tropicalis ATCC13803; C. tropicalis LM45; C. tropicalis LM14; C. tropicalis LM20).

In addition, control of the viability of yeast tested (negative control) and positive control against the antifungal action of nystatin were performed. The assays were conducted in duplicate and incubated at $35^{\circ} \mathrm{C}$ for 48 hours, at approximately $80 \%$ of humidity. The reading of the dilution microplates for MIC determination of test strains was performed by the visual method. To confirm the presence or absence of viable microorganisms in noninhibitory concentrations, a volume of $10 \mu \mathrm{L}$ of TTC dye (2,3,5-triphenyl tetrazolium chloride) was used. This dye is able to reflect the activity of dehydrogenase enzymes involved in the cellular respiration process, which makes it possible to distinguish live samples that are stained in red from dead ones, which maintained the original color [29].

(II) Determination of the Minimum Fungicidal Concentration (MFC). After MIC determination, the concentration corresponding to the inhibitory concentration (MIC) and the two concentrations immediately more concentrated (MICx2 and MICx4) as well as the positive controls were subcultured on Sabouraud dextrose agar (SDA) plates (Difco Laboratories, Detroit plates, Mich., USA) containing $10 \mu \mathrm{L}$ of inoculum. After $24 \mathrm{~h}$ of incubation at $30^{\circ} \mathrm{C}$, the readings of MFCs were performed based on the growth of controls, and the MFC was considered the lowest drug concentration that prevented visible growth of the subculture [28].

Step II: Roughness and Microhardness Test. Consider the following.

(I) Fabrication of Specimens. Thirty heat-polymerized acrylic resin specimens were prepared (Destac Dent, Pirassununga, São Paulo, SP, Brazil) using a silicone matrix of dense condensation (Flexitime, São Paulo, SP, Brazil), measuring $2 \mathrm{~mm}$ in thickness and $4 \mathrm{~mm}$ in diameter.

The specimens were placed in distilled water at $37^{\circ} \mathrm{C}$ for one hour, exposed to dry heat in an oven for 24 hours to remove residual monomer, and polished with carborundum sandpaper (numbers 220, 330, 600, and 1200), felt discs and pumice stone/distilled water paste. Subsequently, they were washed in running water for 30 seconds [30, 31].

(II) Determination of Changes in Surface Roughness and Microhardness. The specimens were divided into three groups ( $n=10)$ : GI: artificial saliva (negative control), obtained from the pharmacy (Table 2), GII: mouthwash with addition of C. zeylanicum (composed of distilled water, Tween 80, and essential oil from C. zeylanicum at MIC), and GIII: nystatin 100,000 UI/mL.

Group I was kept in artificial saliva throughout the test, while groups II and III were immersed for 1 minute, 3 times a day, in their corresponding solutions (mouthwash and nystatin, resp.) and then in artificial saliva for 15 days, during which the system remained incubated in a biological oven in the dark at $37^{\circ} \mathrm{C}$. Initial and final measurements were performed ( 24 hours before and 24 hours after the start and end of the test, resp.) in order to evaluate possible changes in the mechanical properties of the heat-polymerized acrylic resin.

For the roughness trial, the samples received markings on one of the surfaces, corresponding to the middle region, $1 \mathrm{~mm}$ to the right and $1 \mathrm{~mm}$ to the left, and were evaluated (SJ-201 Minutayo, Kawasaki, Japan), according to the ISO 1997 standard (speed $0.5 \mathrm{~mm} / \mathrm{s}, \lambda c 0.8$, and distance 1 ) by which initial and final measurements were performed. For each sample, the arithmetic mean of the three values obtained was calculated, which was considered the surface roughness in micrometers $(\mathrm{Ra})[32,33]$.

For the hardness test, the same samples were used; however, the opposite surface to that intended for the roughness test was measured. Microhardness values were measured before and after the test. A microhardness tester (Shimadzu, Kyoto, Japan) containing a Vickers type diamond tip indenter was used in this step, applying $25 \mathrm{~g}$ of force for 30 seconds. For each specimen, five indentations were performed, and the unit of results was obtained in Vickers hardness number (VHN) after calculating the mean value obtained after five measurements of each sample $[34,35]$.

\subsection{Clinical Stage: Phase I Clinical Trial}

2.4.1. Inclusion and Exclusion Criteria. The study included healthy individuals aged 40-60 years, users of removable full or partial maxillary dentures. Patients who had been under treatment with antimicrobial agents for at least 6 months before the study and those with a history of sensitivity to cinnamon were excluded.

2.4.2. Sample. The sample consisted of 15 patients treated in public service health units. Anamnesis and clinical examinations were performed by two calibrated investigators. $K$ values obtained in the calibration were 0.75 and 1 for inter- and intraexaminer, respectively, representing good and excellent agreement [36].

2.4.3. Clinical and Mycological Diagnosis of Denture Stomatitis. Anamnesis and preliminary clinical examinations were performed to check the absence or presence of signs and symptoms of denture stomatitis, such as change in mucosa texture, presence of whitish areas susceptible to removal by scraping, erythematous mucosa underlying the dental prosthesis, pain, burning, itching, and/or halitosis. Furthermore, a mycological laboratory test was performed by collecting material from the oral mucosa and subsequent cultivation in culture medium. Biological samples (secretion and scales from the palatal mucosa) were collected with a sterile swab, allocated into identified test tubes containing saline $(0.85 \%)$ solution, and sent to the Laboratory of Clinical Mycology, CCS/UFPB, for processing and diagnosis. Sequentially, the samples were grown in culture medium (Chromagar Candida, Difco Laboratories, Detroit, USA) through a calibrated 
loop of $1 \mu \mathrm{L}$ and incubated at room temperature for 48 hours, when the reading was taken.

After obtaining the laboratory results of mycological examination, volunteers with negative diagnosis (clinical and mycological) of candidiasis were recruited and submitted to antifungal therapy, which consisted of administration of a mouthwash based on C. zeylanicum (cinnamon) at MIC, with the following composition: distilled water, essential oil of $C$. zeylanicum, and Tween 80 .

Personal and family history, allergies and hypersensitivities, harmful habits, hygiene habits, and use of drugs, as well as diagnostic information and possible adverse effects, were recorded on a medical record chart before and after the administration of the product. The photographic record of the palate of participants as well as of their dental prostheses was also performed in two time intervals, with the aid of orthodontic mirror and SONY camera (16.1 mega pixels DSC, W570D) in order to serve as an auxiliary tool for qualitative analysis of tolerability and adverse reactions, including the following signs and symptoms: burning, taste alterations, itching, peeling, erythema, and color change of teeth and/or dentures.

2.4.4. Intervention Protocol. Subjects were instructed to use mouthwash 3 times a day for a period of 15 days after cleaning the oral cavity, performing mouth washes with $10 \mathrm{~mL}$ for about 60 seconds, without ingesting the product. They were also informed that, after the use of mouthwash, they should not eat any food and should drink no water for 30 minutes.

The participants received a container containing $500 \mathrm{~mL}$ of test mouthwash, an injector $(10 \mathrm{~mL})$, a stiff bristle tooth brush (Colgate Classic), and toothpaste (Colgate Triple Action) to use for cleaning the dental prostheses. Subsequently, they were also provided with written instructions containing two numbers for contacts with the head researcher in cases of doubt or communication of adverse reaction. In case of the occurrence of unwanted events, the volunteers were instructed to promptly discontinue the intervention protocol and communicate with the head researcher so that undesirable clinical signs and symptoms could be recorded.

During all the days of intervention, two researchers maintained at least one daily verbal contact with participants by phone calls, reminding them to comply with the schedule and of the correct way to use the mouthwash. Twenty-four hours before and after use of the test product, anamnesis and clinical examination were performed accompanied by laboratory mycological examination.

2.5. Statistical Analysis. The database was compiled in the GraphPrisma statistical software program version 6.0. A descriptive statistical analysis of laboratorial clinical and microbiological data was performed. For roughness and hardness trials, ANOVA associated with posttest Tukey test was used, adopting a significance level of $5 \%$.

\section{Results}

The chromatographic analysis of the essential oil from $C$. zeylanicum leaves allowed the identification of seventeen
TABLE 1: Analytes identified by GC-MS in the essential oil from Cinnamomum zeylanicum Blume leaves.

\begin{tabular}{lccc}
\hline Peak & Retention time & Component & Concentration (\%) \\
\hline Peak 1 & 5.961 & $\alpha$-pinene & 0.73 \\
Peak 2 & 6.390 & Camphene & 0.23 \\
Peak 3 & 7.206 & $\beta$-pinene & 0.15 \\
Peak 4 & 8.057 & $\alpha$-phellandrene & 0.52 \\
Peak 5 & 8.747 & $\rho$-pymene & 0.97 \\
Peak 6 & 8.915 & $\beta$-phellandrene & 0.51 \\
Peak 7 & 11.590 & Linalool & 1.92 \\
Peak 8 & 19.118 & (Z)-cinnamaldehyde & 1.04 \\
Peak 9 & 19.934 & Safrole & 0.93 \\
Peak 10 & 23.124 & Eugenol & 82.30 \\
Peak 11 & 23.804 & $\alpha$-copaene & 0.58 \\
Peak 12 & 25.715 & (E)-caryophyllene & 3.33 \\
Peak 13 & 26.752 & $($ E)-cinnamyl acetate & 1.12 \\
Peak 14 & 27.162 & $\alpha$-humulene & 0.50 \\
Peak 15 & 30.222 & Eugenyl acetate & 2.01 \\
Peak 16 & 32.572 & Caryophyllene oxide & 0.25 \\
Peak 17 & 39.486 & Benzyl benzoate & 2.92 \\
\hline Total & \multicolumn{3}{c}{100.00} \\
\hline
\end{tabular}

TABLE 2: Results of MIC and MFC of essential oil from C. zeylanicum leaves and nystatin on Candida species.

\begin{tabular}{lcccc}
\hline \multirow{2}{*}{ Strains } & \multicolumn{2}{c}{ C. zeylanicum } & \multicolumn{2}{c}{ Nystatin } \\
& $\begin{array}{c}\text { MIC } \\
(\mu \mathrm{g} / \mathrm{mL})\end{array}$ & $\begin{array}{c}\text { MFC } \\
(\mu \mathrm{g} / \mathrm{mL})\end{array}$ & $\begin{array}{c}\text { MIC } \\
(\mu \mathrm{g} / \mathrm{mL})\end{array}$ & $\begin{array}{c}\text { MFC } \\
(\mu \mathrm{g} / \mathrm{mL})\end{array}$ \\
\hline C. albicans ATCC 76485 & 312.5 & 625.0 & 32.0 & 128.0 \\
C. albicans ATCC 76645 & 312.5 & 312.5 & 8.0 & 8.0 \\
C. albicans LM P20 & 625.0 & 625.0 & 8.0 & 8.0 \\
C. albicans LM 111 & 625.0 & 625.0 & 8.0 & 8.0 \\
C. albicans LM 62 & 312.5 & 312.5 & 8.0 & 8.0 \\
C. albicans LM 108 & 625.0 & 625.0 & 8.0 & 8.0 \\
C. albicans LM 122 & 625.0 & 625.0 & 8.0 & 8.0 \\
C. albicans LM 86 & 625.0 & 625.0 & 8.0 & 8.0 \\
C. tropicalis ATCC 13803 & 625.0 & 625.0 & 8.0 & 8.0 \\
C. tropicalis LM 45 & 625.0 & 625.0 & 8.0 & 16.0 \\
C. tropicalis LM 14 & 625.0 & 625.0 & 16.0 & 64.0 \\
C. tropicalis LM 20 & 625.0 & 625.0 & 16.0 & 16.0 \\
\hline
\end{tabular}

chromatographic peaks (Table 1 and Figure 1). With regard to the major component, eugenol obtained a relative concentration of $82.30 \%$, with peak at time of 23.3 minutes.

The results of the minimum inhibitory concentration (MIC) and minimum fungicidal concentration (MFC) of the essential oil from C. zeylanicum and nystatin are shown in Table 2.

It was found that all test strains showed sensitivity to the action of the essential oil, and the concentration of $625.0 \mu \mathrm{g} / \mathrm{mL}$ was able to inhibit all samples. Three types of yeast (C. albicans ATCC 76485, C. albicans ATCC 76645, and $C$. albicans LM 62) were inhibited at concentration of 


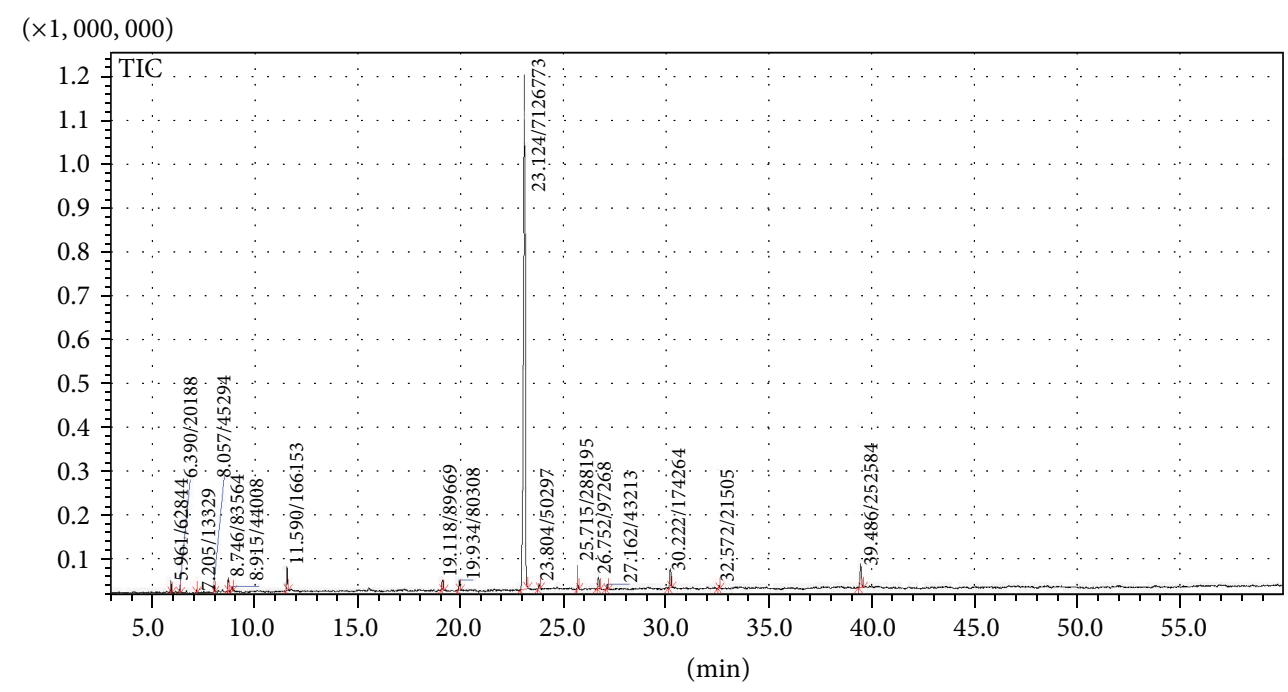

Figure 1: Chromatogram of Cinnamomum zeylanicum essential oil.

$312.5 \mu \mathrm{g} / \mathrm{mL}$, showing the most sensitive behavior to the action of C. zeylanicum.

Among the test strains, eleven showed no microbial growth at a concentration equivalent to that found during MIC determination, equivalent to MFC in $91.66 \%$ of the Candida sample. The only yeast corresponding to C. albicans ATCC 76485 showed MFC equal to MICx2, however, with the value of $625.0 \mu \mathrm{g} / \mathrm{mL}$ remaining as fungicide concentration. It was observed that the concentration of $625 \mu \mathrm{g} / \mathrm{mL}$ was able to prevent microbial growth of all yeast and was also considered the MFC.

Used as a positive control, nystatin also had MIC and MFC values determined (Table 2). Among the test strains, nine demonstrated growth inhibition at concentration of $8 \mu \mathrm{g} / \mathrm{mL}$. Two strains (C. tropicalis LM 14 and C. tropicalis LM 20) were less sensitive to the action of nystatin, with MIC of $16 \mu \mathrm{g} / \mathrm{mL}$, while the sample corresponding to $C$. albicans ATCC 76485 required a higher concentration of the control antifungal agent in order to inhibit its growth (MIC = $32 \mu \mathrm{g} / \mathrm{mL})$.

During the performance of tests to determine the MFC of nystatin, 8 strains equivalent to $66.6 \%$ of the sample showed MFC of $8 \mu \mathrm{g} / \mathrm{mL}$ equivalent to MIC. The same equivalence was observed for yeast C. tropicalis LM 20, with MIC and MFC values being equal to $16 \mu \mathrm{g} / \mathrm{mL}$. C. tropicalis LM 45 and C. tropicalis and LM 14 showed MFC values of $16 \mu \mathrm{g} / \mathrm{mL}$ and $64 \mu \mathrm{g} / \mathrm{mL}$. C. albicans ATCC 76485 showed a higher fungicidal concentration $(128 \mu \mathrm{g} / \mathrm{mL})$, which is 4 times the value of the MIC.

Figure 2 shows the results of mean roughness for GI, GII, and GIII before and after intervention, and no differences were identified between initial measurements of specimens in GI, GII, and GIII $(P>0.05)$. However, significant changes in roughness were observed in the three groups, when their measurements before and after treatment were compared.

In addition to the statistical significance with respect to surface roughness $(\mathrm{Ra})$ between pre- and posttreatment values in the same group, Figure 1 also showed significant

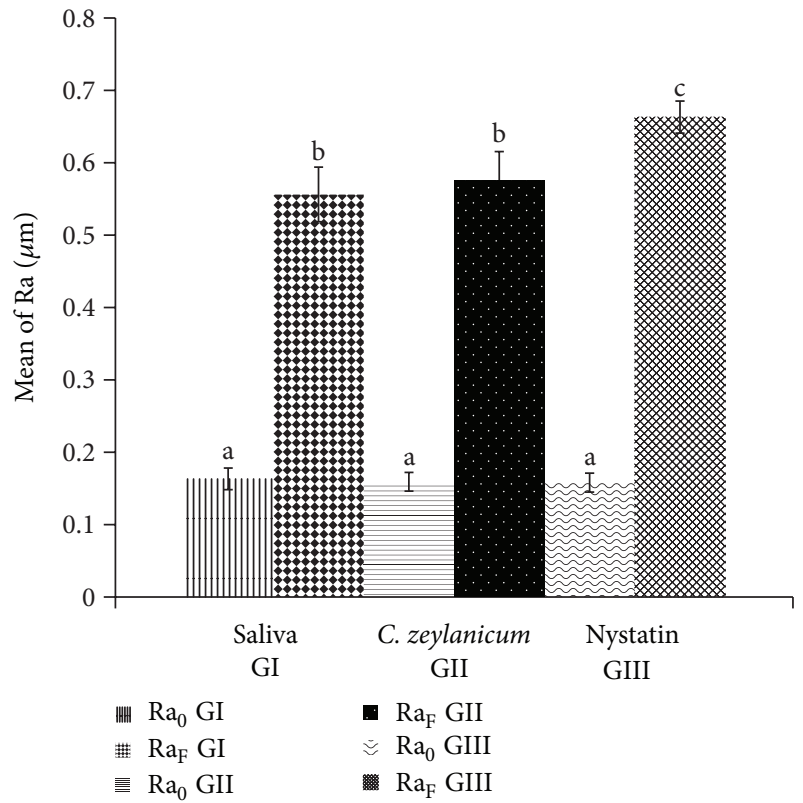

FIgURE 2: Changes in surface roughness $(\mathrm{Ra})$ before and after treatments. $\mathrm{RA}_{0}$ is initial roughness and $\mathrm{Ra}_{\mathrm{F}}$ is final roughness; different letters indicate statistically significant differences, while equal letters indicate absence of statistical significance.

differences in final Ra measures when comparing pairs "GI: saliva/GIII: nystatin" and "GII: C. zeylanicum/GIII: nystatin." It was observed that the negative control (GI: saliva) showed a smaller increase of surface roughness $(0.39 \mu \mathrm{m})$ in comparison with the positive control (GIII: nystatin), in which larger changes occurred $(0.50 \mu \mathrm{m})$ in this property $(P<0.0001)$.

Although the trial of the mouthwash based on C. zeylanicum also showed interference in the surface roughness of samples before and after treatment $(0.40 \mu \mathrm{m})$, these values were lower than those shown for the standard antifungal 


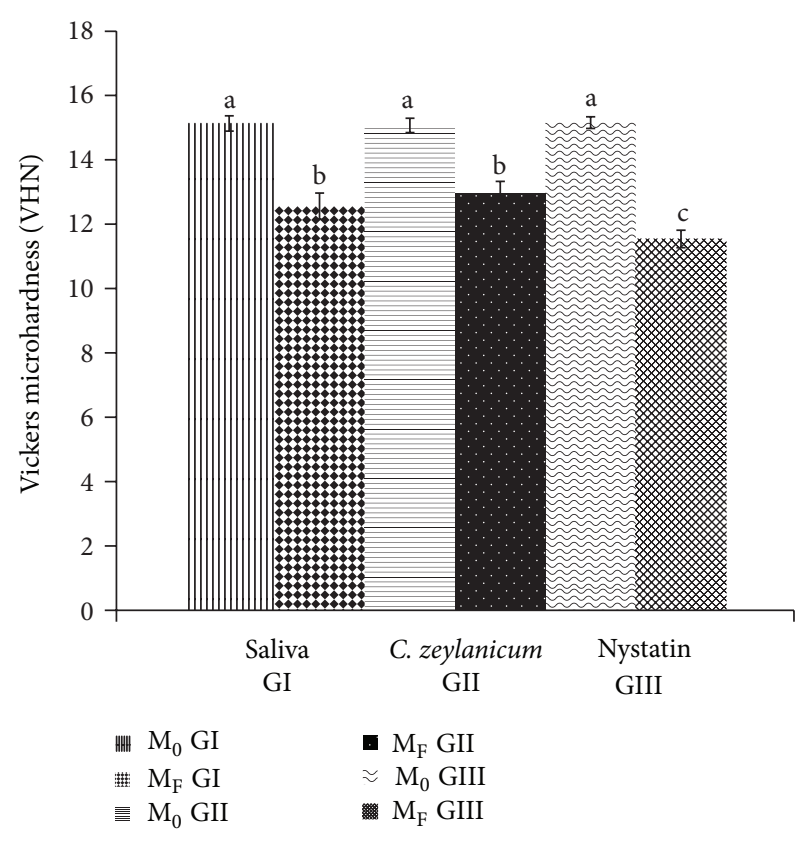

FIGURE 3: Changes in Vickers microhardness before and after treatments. $\mathrm{M}_{0}$ is initial microhardness and $\mathrm{M}_{\mathrm{F}}$ is final microhardness; different letters indicate statistically significant differences, while equal letters indicate absence of statistical significance.

agent, consistent with nystatin intervention $(P<0.0001)$, similar to GI. There was no statistical difference between final roughness measurements $\left(\mathrm{Ra}_{\mathrm{F}}\right)$ and roughness variation $(\Delta \mathrm{Ra})$ of GII (C. zeylanicum) and GI (saliva).

Figure 3 shows the results of the mean microhardness values of five indentations performed for each specimen in GI, GII, and GIII, before and after intervention. No significant differences between baseline values were observed between groups $(P>0.05)$, demonstrating a standardization of the initial samples for the three groups and the minimization of methodological biases. However, there were changes in the three groups $(P<0.0001)$ when initial and final mean values before and after treatment were compared.

It was observed that the groups consisting of the test substance (GII) and saliva (GI) caused a smaller decrease in microhardness (-2.12 VHN and $-2.57 \mathrm{VHN}$, resp.), with no statistical difference between them. It was found, however, that the group consisting of nystatin was capable of producing greater interference in the measured property $(-3.62 \mathrm{VHN})$, which is significant in comparison with the other groups $(P<0.0001)$. These results were positive in comparison with the test substance, since the influence on the decrease in microhardness of the test substance was less than that of nystatin.

\subsection{Results regarding the Phase I Clinical Trial}

3.1.1. Sample Characterization. After conducting the clinical intervention protocol for 15 days, patients were once again submitted to clinical and mycological examination (in which patients were investigated for alteration of palatal mucosa, erythema, edema, milky white plates, and hyperplasia) 24 hours after the end of last mouthrinse. The users were also asked about the presence of any adverse symptoms such as itching, burning, discomfort, loss of taste, and complaints about some modifications in the prosthesis.

After using the test mouthwash, at a concentration of $625 \mu \mathrm{g} / \mathrm{mL}$ (adopted from results obtained in the determination of the minimum inhibitory concentration of $C$. zeylanicum observed in the laboratory phase of this study), none of the participants showed clinical signs of color, shape, or texture change in the area of mucosa that supports the denture. With regard to symptoms, only one of the fifteen patients complained of a slight "burning" in the region of the tongue after using the mouthwash; however, the patient reported that this complaint was of short duration (about 2 minutes after use). On mycological examination, all patients were negative for the growth of fungal colonies on a solid medium.

\section{Discussion}

The genus Cinnamomum includes about 250 species, which are found in nature in the form of shrubs and small to medium sized trees. They can be found in tropical forests, growing in well-drained plains and mountains. The genus is found in abundance in Southeastern East Asia, where species C. zeylanicum, C. loureiroi, C. burmannii, and C. cassia are the most used by the population, and the essential oil obtained from bark and leaves is used as food flavor, in perfumery and also in pharmaceutical preparations [37-39].

The clinical use of natural products with action on oral candidiasis has rarely been reported in literature, and there is a lack of clinical research with essential oil obtained from C. zeylanicum leaves for this purpose. The use of essential oil extracted from the bark of this plant for the treatment of this lesion was described by a single trial, characterized as a pilot study, as it included only five individuals [18]. Essential oils from other plant species have not been studied from a clinical perspective for the treatment of oral candidiasis.

With respect to studies involving the pharmaceutical use or the antimicrobial activity of natural products, it should be stressed that these uses must preferably be associated with a chemical analysis of the test substance [40]. This practice provides the identification of key substances, suggesting a possible biological effect on the microorganisms involved in the disease.

Previous studies have indicated that the essential oil from C. zeylanicum leaf has significant amounts of eugenol [41, 42], corroborating the chromatographic results shown. The antifungal potential of the essential oil from C. zeylanicum bark is associated with high levels of cinnamaldehyde [43] and eugenol $[39,42]$, although other constituents may also contribute to the antimycotic activity.

Evaluation of the effect of phytochemicals (eugenol, cinnamaldehyde, citral, and geraniol) on C. albicans biofilm has shown that their inhibitory activity is dose- and timedependent and is positively influenced by the presence of eugenol [44], which in turn is able to reduce the metabolic 
activity of mature biofilms and inhibit the adhesion of Candida to synthetic surfaces and human epithelial cells [45].

When microorganisms become resistant to therapies adopted, there is an increased incidence of infections and therefore increasing need for constant search for new effective substances [4]. In an attempt to obtain a new alternative treatment for denture stomatitis, laboratory tests of minimum fungicidal inhibitory concentration were performed in this study against twelve Candida strains, eight of them being C. albicans and four being C. tropicalis, because these are the most prevalent in oral fungal infections. These species have specific virulence factors favorable to the formation of biofilms and production of extracellular enzymes such as phospholipases and proteases, capable of promoting the destruction of tissues in the host $[46,47]$.

C. albicans accounted for the largest number of strains selected in this study, which is justified due to its high virulence in comparison with other species. Some studies have suggested that this high virulence is linked to an increased amount of interference in intracellular signaling pathways, such as MAP kinase signal transduction (MitogenActivated Protein). These interferences would generate cellular responses involved in invasive growth, cell wall formation, adaptation to osmotic stress, and reproduction [48].

Other studies have also reported the antifungal activity of the essential oil from C. zeylanicum, even considering the different methodologies and concentrations of the test product [41-43, 49]. As regards nystatin used as a control in this study, there is evidence to prove its antifungal activity [50].

The chemical configuration of the leading molecules identified in the essential oil, especially monoterpenos such as eugenol, gives them hydrophobic properties. This allows their deposition on the lipophilic structures of microorganisms such as the plasma membrane, leading to increased permeability with consequent loss of electrolytes essential to the survival of fungal cells [51]. Other mechanisms of action such as inhibition of membrane synthesis, spore germination, and cell respiration may also be present [52]. It was observed that terpene alcohols such as linalool can act as desiccants and solvents that cause protein denaturation [53].

Acrylic resins have been the materials of choice for the manufacture of dentures since the 1930s, and their use is supported by its properties of hardness, biocompatibility, lack of taste and odor, and roughness, among others [54, 55]. In this sense, it is necessary to evaluate the effect of therapeutic substances that may come into contact with this material during clinical use, such as in the treatment of oral candidiasis associated with the use of dental prostheses. This is the first study to assess the effect of essential oils from C. zeylanicum on the physical properties of the acrylic resin (surface roughness and hardness) used in the manufacture of dentures.

To evaluate the surface roughness of the acrylic resin used in the preparation of dental prostheses, the specimens used in this research were submitted to a process of mechanical polishing and obtained initial mean values of $0.16 \mu \mathrm{m}$ for the three groups. These results are satisfactory, considering that the surface roughness value of heat-polymerized resins can range from $0.03 \mu \mathrm{m}$ to $0.75 \mu \mathrm{m}$ [56]. There was no statistical difference among samples and groups before treatment, revealing a standardization of the initial surface roughness of samples. For final values above $2 \mu \mathrm{m}$, there is a significant increase in the colonization of microorganisms on the surface of the acrylic resin [57].

In the present study, there was a significant increase in surface roughness for all groups, including the negative control group (saliva), for which the specimens remained immersed in artificial saliva for fifteen days. The significant increase in surface roughness in the control group can be attributed to sorption and solubility during the immersion process, which has a direct influence on the mechanical properties of the heat-polymerized acrylic resin [58].

Although all groups underwent significant changes in surface roughness in their final measures, there was no significant difference between final roughness of the saliva and test product groups, which had a lower surface roughness change compared with the reference group (nystatin). These results generate positive perspectives for a possible clinical use of mouthwash based on C. zeylanicum in further trials.

In order to draw a tolerability profile of the product used, the property with regard to Vickers microhardness was also evaluated for the three groups. Hardness can be defined as the ability of a substance to resist the wear or penetration. Knowledge of the hardness of materials used in dentistry is very important since it is related to the clinical longevity of materials. The hardness test is a nondestructive, specifically located laboratory test, which provides data on the distribution of properties of materials [59].

In this study, specimens showed no statistical differences in initial mean values among groups for Vickers microhardness, suggesting sample standardization. However, there was significant decrease in this property for the three groups after intervention. Although differences were observed in the final measures among groups, in which the mouthwash based on C. zeylanicum caused a lower degree of change in hardness when compared with the reference group nystatin, these results demonstrate that although causing changes in the Vickers hardness of the material, these changes were of a lower degree than those caused by the standard product (nystatin) used in clinical practice, thus encouraging the possibility of further studies that promote the suggestion of the test product at MIC, as a tolerable alternative for therapeutic use.

Previous studies $[60,61]$ have found a significant reduction in the microhardness of the acrylic resin when using other substances (sodium hypochlorite, $4 \%$ chlorhexidine gluconate, $2 \%$ glutaraldehyde, deionized water, and distilled water) with the aim of disinfecting this material, possibly suggesting that immersion causes changes in the mechanical properties of the material due to the occurrence of sorption and solubility during cycles.

Toxicological studies and phase I clinical trials involving natural products are considered scarce in dentistry, given their importance, so that the scientific evidence shown by in vitro assays is able to propose new therapeutic possibilities [62]. 
The use of natural products in specific situations, such as in dermatology, can often be risky, because they may cause dermatosis due to plants resulting from mechanisms of irritation or photosensitivity [63]. This suggests the importance of conducting a phase I clinical trial for therapies involving the use of natural products, such as that proposed in the present study, in spite of the widespread and well-known use of cinnamon by populations, in the form of spice and flavoring.

For the phase I clinical trial, a sample of 15 subjects was adopted, which was determined from the design proposed for phase I clinical trials involving a small group of healthy volunteers in order to propose or establish a preliminary evaluation of the tolerability of a test product [27].

The results of phase I clinical trial demonstrated a good perspective for the advancement of phase II clinical trials involving C. zeylanicum at MIC of $625 \mu \mathrm{g} / \mathrm{mL}$, since no patient had clinical changes in the mucosa near the dental prosthesis or in other regions of the oral mucosa. As regards symptoms, only one patient reported burning of mild intensity and short duration, and this result is considered satisfactory, given the absence of clinical signs suggestive of any type of acute changes such as erythema or hyperemia, compatible with such symptoms.

Therefore, in view of the foregoing, above and considering the limitations of laboratory steps shown in this research, the study considered the safety and tolerability of the test substance satisfactory, supporting new trials involving the use of essential oil from C. zeylanicum in subsequent clinical stages of scientific evidence.

\section{Conclusion}

The essential oil, obtained from C. zeylanicum leaves, has activity against Candida species, promotes physical changes (roughness and hardness) in the acrylic resin similar to artificial saliva, and presents safety and tolerability when used by healthy individuals subsidizing the development of phase II clinical trial required for the therapeutic investigation of this product in individuals with candidiasis associated with the use of dentures.

\section{Conflict of Interests}

The authors declare that there is no conflict of interests regarding the publication of this paper.

\section{References}

[1] E. Martori, R. Ayuso-Montero, J. Martinez-Gomis, M. Viñas, and M. Peraire, "Risk factors for denture-related oral mucosal lesions in a geriatric population," Journal of Prosthetic Dentistry, vol. 111, no. 4, pp. 273-279, 2014.

[2] N. Navabi, A. Gholamhoseinian, B. Baghaei, and M. A. Hashemipour, "Risk factors associated with denture stomatitis in healthy subjects attending a dental school in Southeast Iran," Sultan Qaboos University Medical Journal, vol. 13, no. 4, pp. 574580, 2013.
[3] A. Jainkittivong, V. Aneksuk, and R. P. Langlais, "Oral mucosal lesions in denture wearers," Gerodontology, vol. 27, no. 1, pp. 2632, 2010.

[4] P. C. Anibal, I. C. O. Saroili, I. T. A. Peixoto, J. J. C. Moraes, and J. F. Hoffling, "Conventional and alternative antifungal therapies to oral candidiasis," Brazilian Journal of Microbiology, vol. 41, no. 4, pp. 824-831, 2010.

[5] L. Gendreau and Z. G. Loewy, "Epidemiology and etiology of denture stomatitis," Journal of Prosthodontics, vol. 20, no. 4, pp. 251-260, 2011.

[6] E. Uzunoglu, A. Z. Y. Bicer, I. Dolapci, and A. Dogan, "Biofilmforming ability and adherence to poly-(methyl-methacrylate) acrylic resin materials of oral Candida albicans strains isolated from HIV positive subjects," Journal of Advanced Prosthodontics, vol. 6, no. 1, pp. 30-34, 2014.

[7] S. Jackson, L. Coulthwaite, Z. Loewy, A. Scallan, and J. Verran, "Biofilm development by blastospores and hyphae of Candida albicans on abraded denture acrylic resin surfaces," Journal of Prosthetic Dentistry, vol. 112, no. 4, pp. 988-993, 2014.

[8] D. Jain and P. Shakya, "An in vitro study on effect of Delmopinol application on Candida albicans adherence on heat cured denture base acrylic resin: a thorough study," Indian Journal of Dental Research, vol. 24, no. 5, pp. 645-646, 2013.

[9] E. Emami, M. Kabawat, P. H. Rompre, and J. S. Feine, "Linking evidence to treatment for denture stomatitis: a meta-analysis of randomized controlled trials," Journal of Dentistry, vol. 42, no. 2, pp. 99-106, 2014.

[10] J. A. Skupien, F. Valentini, N. Boscato, and T. Pereira-Cenci, "Prevention and treatment of Candida colonization on denture liners: a systematic review," Journal of Prosthetic Dentistry, vol. 110, no. 5, pp. 356-362, 2013.

[11] S. Paul and W. S. Moye-Rowley, "Multidrug resistance in fungi: regulation of transporter-encoding gene expression," Frontiers in Physiology, vol. 5, article 143, 2014.

[12] M. E. Da Silva Ferreira, A. L. Colombo, I. Paulsen et al., "The ergosterol biosynthesis pathway, transporter genes, and azole resistance in Aspergillus fumigatus," Medical Mycology, vol. 43, no. 1, pp. 313-319, 2005.

[13] S. J. Doddanna, S. Patel, M. A. Sundarrao, and R. S. Veerabhadrappa, "Antimicrobial activity of plant extracts on Candida albicans: an in vitro study," Indian Journal of Dental Research, vol. 24, no. 4, pp. 401-405, 2013.

[14] J. F. Höfling, P. C. Anibal, G. A. Obando-Pereda et al., "Antimicrobial potential of some plant extracts against Candida species," Brazilian Journal of Biology, vol. 70, no. 4, pp. 10651068, 2010.

[15] P. V. Rao and S. H. Gan, "Cinnamon: a multifaceted medicinal plant," Evidence-Based Complementary and Alternative Medicine, vol. 2014, Article ID 642942, 12 pages, 2014.

[16] P. Ranasinghe, R. Jayawardana, P. Galappaththy, G. R. Constantine, N. de Vas Gunawardana, and P. Katulanda, "Efficacy and safety of "true" cinnamon (Cinnamomum zeylanicum) as a pharmaceutical agent in diabetes: a systematic review and metaanalysis," Diabetic Medicine, vol. 29, no. 12, pp. 1480-1492, 2012.

[17] P. Ranasinghe, S. Pigera, G. S. Premakumara, P. Galappaththy, G. R. Constantine, and P. Katulanda, "Medicinal properties of "true" cinnamon (Cinnamomum zeylanicum): a systematic review," BMC Complementary and Alternative Medicine, vol. 13, article 275, 2013.

[18] J. M. Quale, D. Landman, M. M. Zaman, S. Burney, and S. S. Sathe, "In vitro activity of Cinnamomum zeylanicum against 
azole resistant and sensitive Candida species and a pilot study of cinnamon for oral candidiasis," The American Journal of Chinese Medicine, vol. 24, no. 2, pp. 103-109, 1996.

[19] C. W. Gayoso, E. O. Lima, V. T. Oliveira et al., "Sensitivity of fungi isolated from onychomycosis to Eugenia cariophyllata essential oil and eugenol," Fitoterapia, vol. 76, no. 2, pp. 247-249, 2005.

[20] R. D. de Castro and E. O. Lima, "Anti-candida activity and chemical composition of Cinnamomum zeylanicum blume essential oil," Brazilian Archives of Biology and Technology, vol. 56, no. 5, pp. 749-755, 2013.

[21] I. Pellegatti Lemonica and A. M. R. Borro Macedo, "Abortive and/or embryofetotoxic effect of Cinnamomum zeylanicum leaf extracts in pregnant rats," Fitoterapia, vol. 13, no. 1, pp. 431-434, 1994.

[22] A. Eidi, P. Mortazavi, M. Bazargan, and J. Zaringhalam, "Hepatoprotective activity of cinnamon ethanolic extract against CCL 4-induced liver injury in rats," EXCLI Journal, vol. 11, pp. 495-507, 2012.

[23] E. R. Kitazuru, A. V. B. Moreira, J. Mancini-Filho, H. Delincée, and A. L. C. H. Villavicencio, "Effects of irradiation on natural antioxidants of cinnamon (Cinnamomum zeylanicum N.)," Radiation Physics and Chemistry, vol. 71, no. 1-2, pp. 37-39, 2004.

[24] S. Chericoni, J. A. Prieto, P. Iacopini, P. Cioni, and I. Morelli, "In vitro activity of the essential oil of Cinnamomum zeylanicum and eugenol in peroxynitrite-induced oxidative processes," Journal of Agricultural and Food Chemistry, vol. 53, no. 12, pp. 4762-4765, 2005.

[25] A. J. Zissis, G. L. Polyzois, S. A. Yannikakis, and A. Harrison, "Roughness of denture materials: a comparative study," International Journal of Prosthodontics, vol. 13, no. 2, pp. 136-140, 2000.

[26] V. N. Trajano, E. D. O. Lima, A. E. Travassos, and E. L. de Souza, "Inhibitory effect of the essential oil from Cinnamomum zeylanicum Blume leaves on some food-related bacteria," Ciencia e Tecnologia de Alimentos, vol. 30, no. 3, pp. 771-775, 2010.

[27] L. M. Friedman, C. D. Furberg, and D. L. DeMets, Fundamentals of Clinical Trials, Springer, New York, NY, USA, 4th edition, 2010.

[28] J. N. Eloff, "A sensitive and quick microplate method to determine the minimal inhibitory concentration of plant extracts for bacteria," Planta Medica, vol. 9, no. 2, pp. 392-397, 1998.

[29] D. P. Deswal and V. Chand, "Standartization of the tethazolium test for viability estimation in recebean (Vigna umbellata T.) seeds," Seed Science and Technology, vol. 25, no. 1, pp. 409-417, 1997.

[30] R. C. M. M. Rodrigues Garcia, J. A. de Souza Jr., R. N. Rached, and A. A. del Bel Cury, "Effect of denture cleansers on the surface roughness and hardness of a microwave-cured acrylic resin and dental alloys," Journal of Prosthodontics, vol. 13, no. 3, pp. 173-178, 2004.

[31] International Standards Organization, "ISSO 1567:1999/Amd 1:2003, Dentistry-denture base polymers," Amendment, http:// www.iso.ch/iso/en/prods-services/ISOstore/store.html.

[32] H. Nagem Filho, M. T. F. S. D’Azevedo, H. D. Nagem, and F. P. Marsola, "Surface roughness of composite resins after finishing and polishing," Brazilian Dental Journal, vol. 14, no. 1, pp. 37-41, 2003.

[33] E. A. Sartori, C. B. Schmidt, L. F. Walber, and R. S. A. Shinkai, "Effect of microwave disinfection on denture base adaptation and resin surface roughness," Brazilian Dental Journal, vol. 17, no. 3, pp. 197-200, 2006.
[34] R. R. Regis, N. C. Soriani, A. M. Azevedo, C. H. Silva-Lovato, H. F. Oliveira Paranhos, and R. F. De Souza, "Effects of ethanol on the surface and bulk properties of a microwave-processed PMMA denture base resin," Journal of Prosthodontics, vol. 18, no. 6, pp. 489-495, 2009.

[35] N. H. Campanha, A. C. Pavarina, J. H. Jorge, C. E. Vergani, A. L. MacHado, and E. T. Giampaolo, "The effect of long-term disinfection procedures on hardness property of resin denture teeth," Gerodontology, vol. 29, no. 2, pp. e571-e576, 2012.

[36] J. R. Landis and G. G. Koch, "The measurement of observer agreement for categorical data," Biometrics, vol. 33, no. 1, pp. 159-174, 1977.

[37] I. B. Jantan, N. Ayop, A. B. Hiong, and A. S. Ahmad, "Chemical composition of the essential oils of Cinnamomum cordatum Kosterm," Flavour and Fragrance Journal, vol. 17, no. 3, pp. 212 214, 2002.

[38] I. bin Jantan, N. Ayop, N. A. M. Ali et al., "The essential oils of Cinnamomum rhyncophyllum Miq. as natural sources of benzyl benzoate, safrole and methyl (E)-cinnamate," Flavour and Fragrance Journal, vol. 19, no. 3, pp. 260-262, 2004.

[39] I. B. Jantan, B. A. Karim Moharam, J. Santhanam, and J. A. Jamal, "Correlation between chemical composition and antifungal activity of the essential oils of eight Cinnamomum species," Pharmaceutical Biology, vol. 46, no. 6, pp. 406-412, 2008.

[40] D. L. Nelson and M. M. Cox, Lehninger Principles of Biochemistry, W. H. Freeman, New York, NY, USA, 6th edition, 2013.

[41] P. Pozzatti, L. A. Scheid, T. B. Spader, M. L. Atayde, J. M. Santurio, and S. H. Alves, "In vitro activity of essential oils extracted from plants used as spices against fluconazoleresistant and fluconazole-susceptible Candida spp," Canadian Journal of Microbiology, vol. 54, no. 11, pp. 950-956, 2008.

[42] L. S. M. Ooi, Y. Li, S.-L. Kam, H. Wang, E. Y. L. Wong, and V. E. C. Ooi, "Antimicrobial activities of Cinnamon oil and cinnamaldehyde from the Chinese medicinal herb Cinnamomum cassia Blume," The American Journal of Chinese Medicine, vol. 34, no. 3, pp. 511-522, 2006.

[43] A. Khan, A. Ahmad, F. Akhtar et al., "Induction of oxidative stress as a possible mechanism of the antifungal action of three phenylpropanoids," FEMS Yeast Research, vol. 11, no. 1, pp. 114122, 2011.

[44] S. B. de Paula, T. F. Bartelli, V. Di Raimo et al., "Effect of eugenol on cell surface hydrophobicity, adhesion, and biofilm of Candida tropicalis and Candida dubliniensis isolated from oral cavity of HIV-infected patients," Evidence-Based Complementary and Alternative Medicine, vol. 2014, Article ID 505204, 8 pages, 2014.

[45] M. Unlu, E. Ergene, G. V. Unlu, H. S. Zeytinoglu, and N. Vural, "Composition, antimicrobial activity and in vitro cytotoxicity of essential oil from Cinnamomum zeylanicum Blume (Lauraceae)," Food and Chemical Toxicology, vol. 48, no. 11, pp. 32743280, 2010.

[46] S. C. Deorukhkar, S. Saini, and S. Mathew, "Virulence factors contributing to pathogenicity of Candida tropicalis and its antifungal susceptibility profile," International Journal of Microbiology, vol. 2014, Article ID 456878, 6 pages, 2014.

[47] S. C. Deorukhkar and S. Saini, "Non albicans Candida species: its isolation pattern, species distribution, virulence factors and antifungal susceptibility profile," International Journal of Medicine and Public Health, vol. 2, no. 3, pp. 533-538, 2013. 
[48] R. A. Monge, E. Román, C. Nombela, and J. Pla, "The MAP kinase signal transduction network in Candida albicans," Microbiology, vol. 152, no. 4, pp. 905-912, 2006.

[49] R. D. Castro and E. O. Lima, "Antifungal activity of Brazilian sassafras (Ocotea odorifera Vell.) and rosemary (Rosmarinus officinalis L.) essential oils against the genus Candida," Revista Brasileira de Plantas Medicinais, vol. 13, no. 2, pp. 203-208, 2011.

[50] S. M. Gunderson, H. Hoffman, E. J. Ernst, M. A. Pfaller, and M. E. Klepser, "In vitro pharmacodynamic characteristics of nystatin including time-kill and postantifungal effect," Antimicrobial Agents and Chemotherapy, vol. 44, no. 10, pp. 2887-2890, 2000.

[51] F. Bakkali, S. Averbeck, D. Averbeck, and M. Idaomar, "Biological effects of essential oils-a review," Food and Chemical Toxicology, vol. 46, no. 2, pp. 446-475, 2008.

[52] R. Harris, "Progress with superficial mycoses using essential oils," International Journal of Aromatherapy, vol. 12, no. 2, pp. 83-91, 2002.

[53] D. H. J. Dorman, "In vitro evaluation of antioxidant activity of essential oils their components," Flavour and Fragrance Journal, vol. 15, no. 1, pp. 12-16, 2000.

[54] F. A. Peyton, "History of resins in dentistry," Dental Clinics of North America, vol. 19, no. 2, pp. 211-222, 1975.

[55] American Dental Association, "Specification no 12 for denture base polymers. Councils on Dent Materials and Bureaus," Journal of the American Dental Association, vol. 90, pp. 39-49, 1975.

[56] J. S. Rahal, M. F. Mesquita, G. E. P. Henriques, and M. A. A. Nóbilo, "Surface roughness of acrylic resins submitted to mechanical and chemical polishing," Journal of Oral Rehabilitation, vol. 31, no. 11, pp. 1075-1079, 2004.

[57] L. M. J. Mímica, S. M. Y. Ueda, M. D. V. Martino, A. Navarini, and I. J. Martini, "Candida infection diagnosis: evaluation of Candida species identification and characterization of susceptibility profile," Jornal Brasileiro de Patologia e Medicina Laboratorial, vol. 5, no. 1, pp. 17-23, 2009.

[58] H. D. F. O. Paranhos, A. Peracini, M. X. Pisani, V. D. C. Oliveira, R. F. D. souza, and C. H. Silva-Lovato, "Color stability, surface roughness and flexural strength of an acrylic resin submitted to simulated overnight immersion in denture cleansers," Brazilian Dental Journal, vol. 24, no. 2, pp. 152-156, 2013.

[59] A. P. Farina, D. Cecchin, R. G. Soares et al., "Evaluation of Vickers hardness of different types of acrylic denture base resins with and without glass fibre reinforcement," Gerodontology, vol. 29, no. 2, pp. e155-e160, 2012.

[60] L. de Rezende Pinto, E. J. T. R. Acosta, F. F. F. Távora, P. M. B. Da Silva, and V. C. Porto, "Effect of repeated cycles of chemical disinfection on the roughness and hardness of hard reline acrylic resins," Gerodontology, vol. 27, no. 2, pp. 147-153, 2010.

[61] R. R. Regis, N. C. Soriani, A. M. Azevedo, C. H. Silva-Lovato, H. F. O. Paranhos, and R. F. de Souza, "Effects of ethanol on the surface and bulk properties of a microwave-processed PMMA denture base resin," Journal of Prosthodontics, vol. 18, no. 6, pp. 489-495, 2009.

[62] V. F. Veiga Jr., A. C. Pinto, and M. A. M. Maciel, "Medicinal plants: safe cure?" Quimica Nova, vol. 28, no. 3, pp. 519-528, 2005.

[63] V. M. S. Reis, "Dermatosis due to plants (phytodermatosis)," Anais Brasileiros de Dermatologia, vol. 85, no. 4, pp. 479-484, 2010. 


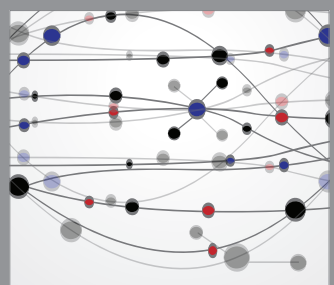

The Scientific World Journal
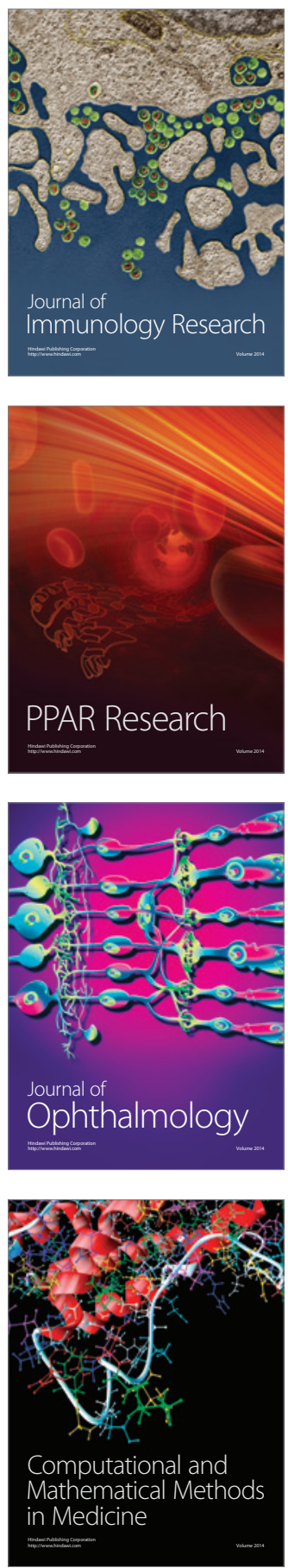

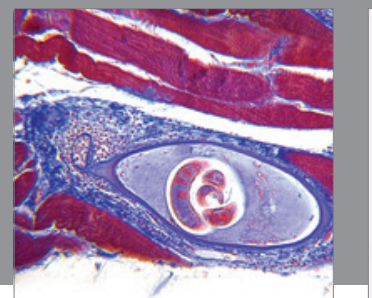

Gastroenterology

Research and Practice
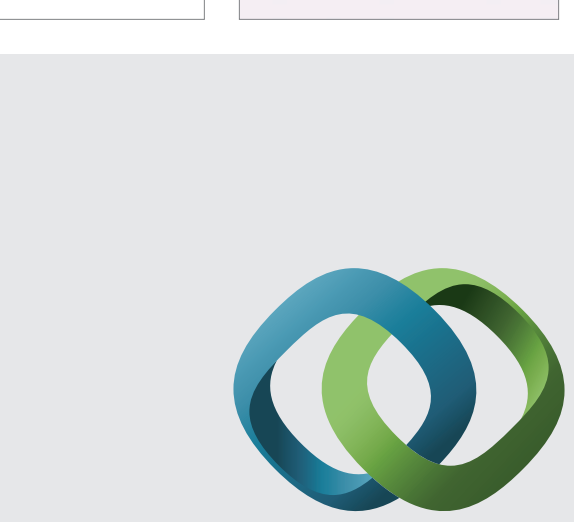

\section{Hindawi}

Submit your manuscripts at

http://www.hindawi.com
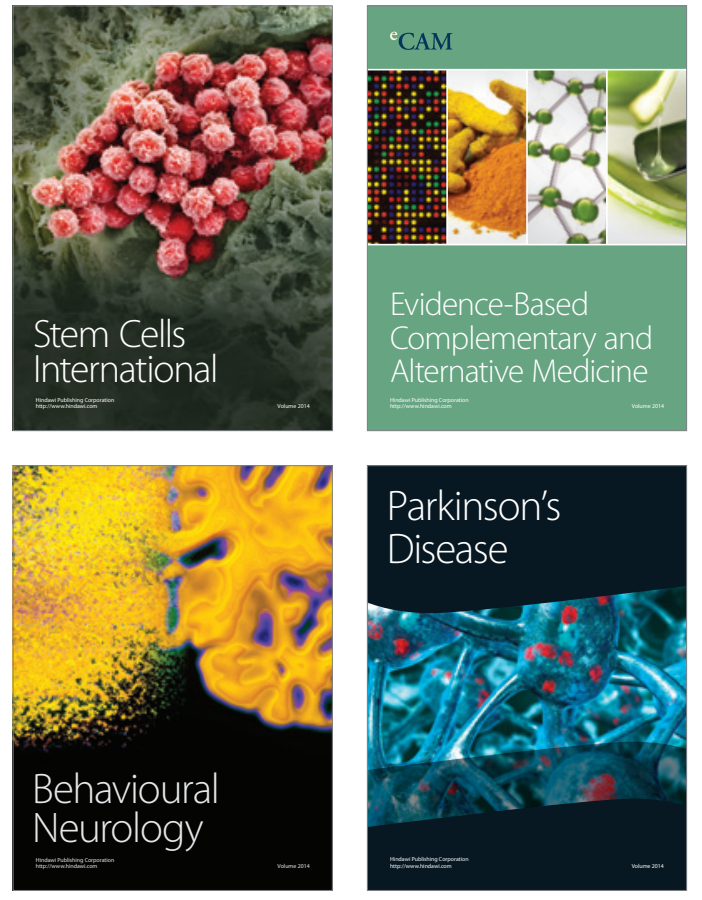
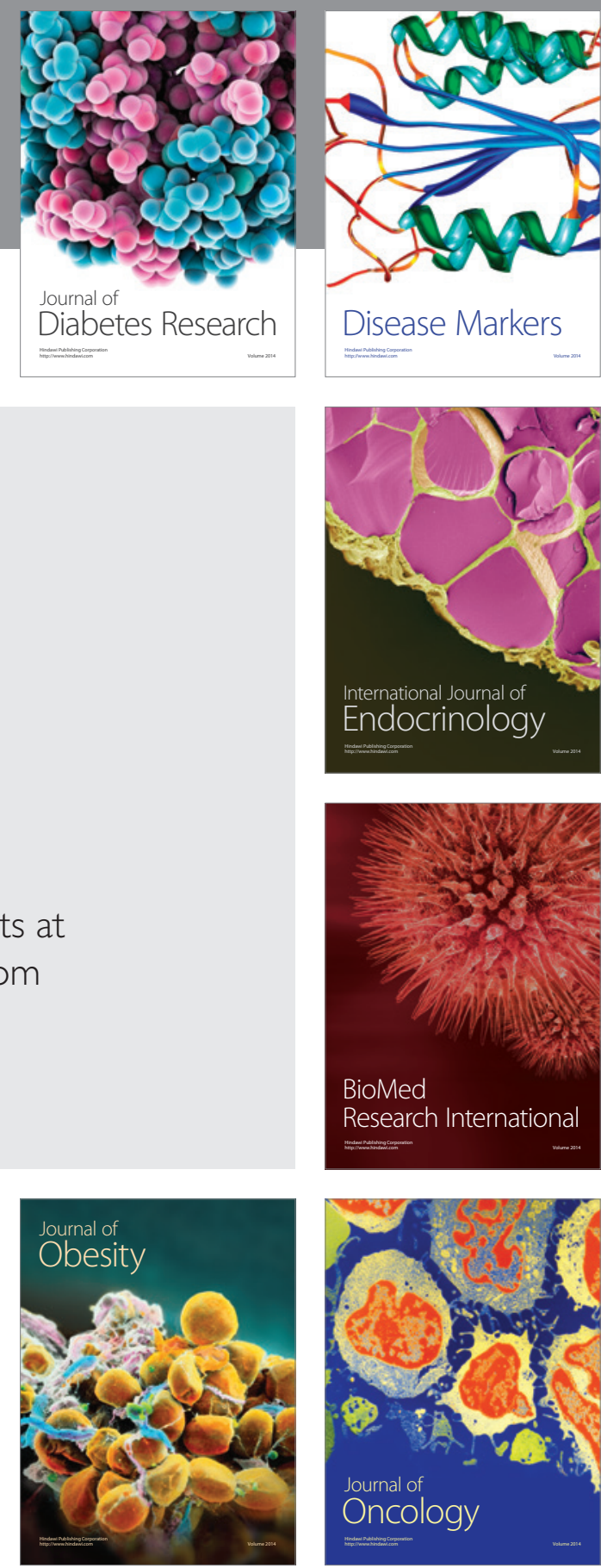

Disease Markers
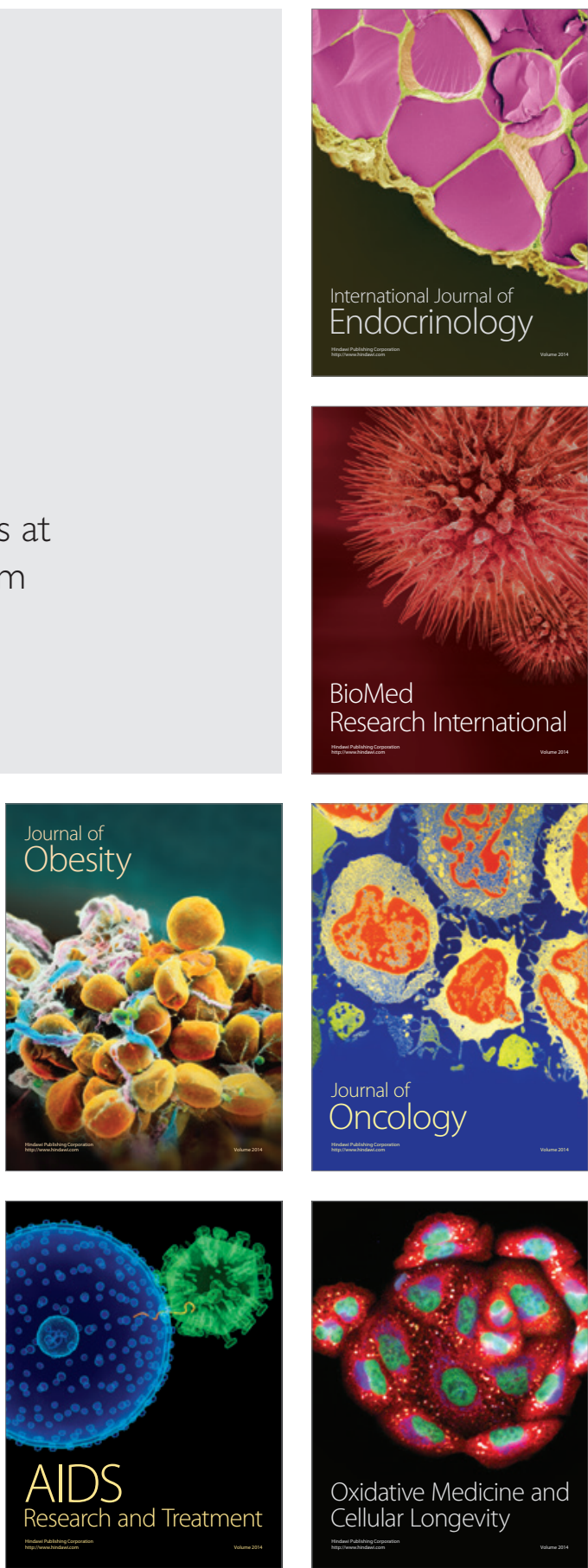